\title{
Vi vogliamo dissuadere o persuadere?
}

\section{Rossella Varvara}

PUBBLICATO: 03 MAGGIO 2019

\section{Quesito:}

In molti ci chiedono chiarimenti sui verbi dissuadere e persuadere. I lettori ci domandano dove sia corretto porre l'accento, quale sia la differenza di significato tra i due termini e quali preposizioni introducono i loro complementi.

\section{Vi vogliamo dissuadere o persuadere?}

a domanda più frequente che ci è stata posta a proposito dei verbi dissuadere e persuadere riguarda la loro pronuncia, ovvero la posizione dell'accento tonico. Ebbene, entrambi i verbi sono piani, cioè accentati sulla penultima sillaba, come riportato da qualsiasi dizionario italiano. Diremo quindi dissuadére e persuadére, e non dissuádere o persuádere. Lo Zingarelli 2018 consiglia di evitare queste ultime pronunce, dovute probabilmente all'influsso di alcune forme del presente indicativo (persuádo, dissuádono). Lo stesso vale per le forme dellinfinito seguite dai pronomi atoni (le cosiddette "particelle pronominali": mi, ti, ci, vi, ecc.): diremo dissuadérlo e non dissuáderlo.

Come per molte altre parole italiane, l'accentazione piana è un'eredità del latino. Per le parole di tre sillabe, in latino l'accento era determinato dalla natura della penultima sillaba: con una sillaba breve (ovvero, che non terminava in consonante e che conteneva una vocale breve) l'accento indietreggiava sulla terzultima sillaba, come in portǐcus 'portico', pronunciato pórticus; l'accento invece restava sulla penultima sillaba se questa era lunga, cioè se conteneva una vocale lunga (come nel caso dei verbi latini dissuadère e persuadère) oppure se era "implicata", cioè terminava in consonante (come per perfëctum, pronunciato perféctum).

L'etimologia ci rivela dunque la corretta pronuncia di questi verbi e ci ricorda anche la loro vicinanza nella forma e nel significato. Persuadere e dissuadere derivano dal verbo latino suadère 'consigliare, persuadere' a cui si aggiunsero i due prefissi per- e dis-. Il prefisso per- 'fino in fondo' rafforzava il significato del verbo base; dis-, invece, aggiungeva un valore negativo (come anche ad es. in disonore) o indicava separazione, dispersione (disgiungere, distrarre). Dissuadere, quindi, era ed è tutt'oggi il corrispettivo negativo di persuadere. Useremo persuadere quando vogliamo convincere qualcuno di qualcosa o a fare una determinata azione; dissuadere per convincerlo a non farla. Possiamo dire, ad esempio, che Giovanni ha persuaso Luigi a non comprare quella casa e, preservando lo stesso significato, che Giovanni ha dissuaso Luigi dal comprare quella casa.

Come mostrano questi esempi, i due verbi selezionano preposizioni diverse per introdurre il complemento indiretto. Il verbo dissuadere richiede la preposizione da (anche nella forma articolata) per introdurlo, sia esso un nome (es. I) o una proposizione, cioè una frase subordinata, infinitiva (es. 2; tutti gli esempi, là dove non diversamente specificato, sono tratti dal Corpus la Repubblica):

I. i suoi consiglieri più fidati lo avrebbero dissuaso da questo proposito

2. hanno cercato di dissuaderlo dal continuare lo sciopero 
In passato, oltre ad attestazioni con la preposizione da (ad esempio la frase del passo 3 tratta dall'Orlando Furioso dell'Ariosto e citata dal Tommaseo-Bellini) o con la preposizione di (esempio in 4), era piuttosto comune esprimere ciò da cui si vuol distogliere qualcuno anche con un complemento diretto, come nei passi 5 e 6 citati dal GDLI, possibilità tuttavia attualmente non più accettabile. In questi casi, la persona sulla cui volontà si voleva intervenire era indicata da un complemento di termine, come nel passo 5 .

I. Ma il Re Sobrino, il quale era presente, / Da questa impresa molto il dissuade. (Ludovico Ariosto, Orlando Furioso, Canto 27)

2. Prima di annottare, una o due poste prima di Bovino, l'oste ed il conduttore cercarono di dissuaderlo di andare innanzi, perché la campagna era infestata dai briganti (Giovanni Verga, Certi argomenti, 1877).

3. Scrive al collegio de' cardinali, confortandoli a dissuadere al pontefice la discordia tra il sacerdozio e l'imperio (Pandolfo Collenuccio, Compendio de le istorie del regno di Napoli, I929).

4. I generali austriaci, non soliti a commettersi all'arbitrio della fortuna, dissuadevano la battaglia (Carlo Botta, Storia d'Italia dal I789 al I8I4, I839).

Sebbene la preposizione da sia l'unica ammessa dai dizionari, oggi si attesta qualche uso con l'infinitiva introdotta anche dalla preposizione $a$. Nel Corpus la Repubblica, ad esempio, sono almeno dieci le occorrenze di dissuadere seguito da $a+$ infinito.

5. il terrorismo mediorientale personalizzato sul bersaglio Yankee per quanto minaccioso non lo è al punto dal dissuaderlo a frequentare il nostro paese

6. L' ingorgo di scadenze dissuade a pagare subito

Questi casi sono probabilmente dovuti alla confusione con il verbo persuadere, di cui dissuadere come si è visto è l'opposto.

Persuadere, infatti, può reggere una frase infinitiva introdotta sia da questa preposizione sia dalla preposizione di:

7. Due deputati gli sono corsi incontro per persuaderlo a cambiare idea

8. Gertrude di Helfta finisce col persuadersi di essere lei stessa la madre del Cristo

Con un sintagma nominale, invece, la preposizione utilizzata è soltanto di, anche nella forma articolata:

I. Sono persuaso del valore del libro

2. Personalmente non sono persuaso di questa scelta

Tornando ai casi con una frase subordinata infinitiva, possiamo chiederci quale sia il rapporto di distribuzione tra le due preposizionidi e a. Le due preposizioni non sembrano, infatti, interscambiabili, come possiamo osservare invertendole negli esempi g e ro nelle frasi seguenti:

9. *Due deputati gli sono corsi incontro per persuaderlo di cambiare idea

Io. *Gertrude di Helfta finisce col persuadersi a essere lei stessa la madre del Cristo

L'asterisco a inizio frase indica in linguistica la non correttezza della frase. L'esempio iz non è accettabile con la preposizione di, mentre in I4 è la preposizione $a$ a rendere la frase scorretta. 
Possiamo individuare tre differenze nell'uso di queste due costruzioni di persuadere. Diversi linguisti (Skytte, I983, Skytte e Salvi I99I, Lombardi Vallauri, 20II) hanno osservato come, in primo luogo, ci sia una differenza di significato tra il verbo persuadere con le due diverse preposizioni. Nel caso di $a$, persuadere ha il senso di 'convincere a fare', mentre con di il suo significato è parafrasabile con 'convincere a credere', o semplicemente 'credere, far credere'.

In secondo luogo, quando è seguito dalla preposizione $a$, l'infinito si riferisce a un'azione futura, che deve ancora avvenire, mentre con la preposizione di tale connotazione di futuro è esclusa. L'azione dell'infinito è in questo caso contemporanea o anteriore a quella della principale.

Si osservi la differenza di significato che otteniamo nelle frasi seguenti cambiando semplicemente la preposizione:

I. persuaso di amare sempre Marion, il romantico Pierre si comporta come uno stordito

2. persuaso ad amare sempre Marion, il romantico Pierre si comporta come uno stordito

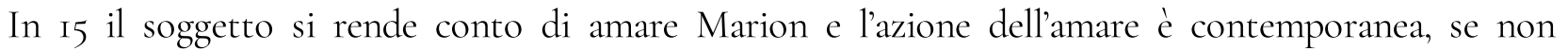
anteriore, al persuardersi. L'avverbio sempre sembra in questo caso interpretabile come un 'ancora'. In I6 invece il cambio di preposizione ci lascia desumere che il soggetto intende amare Marion, si è convinto ad amarla, ma l'amarla è un atto futuro.

Come ulteriore prova di questa differenza temporale, si può notare come con la preposizione $a$, contrariamente a quanto accade con di, non sia accettabile l'infinito passato:

3. Poi, persuaso d'averla detta grossa, s'affretta ad aggiungere

4. *Poi, persuaso ad averla detta grossa, s'affretta ad aggiungere

Infine, da un punto di vista sintattico, la frase subordinata introdotta da di puó essere una preposizione soggettiva, in quanto il soggetto dell'infinito può corrispondere al soggetto del verbo della principale. Nella frase seguente, il soggetto dell'infinitiva è Gianni, ovvero il soggetto della principale:

3. Gianni ha persuaso Luigi di essere sincero

Con la preposizione $a$, invece, l'infinitiva è oggettiva, ovvero il soggetto dell'infinito coincide con il complemento oggetto della principale:

4. Gianni ha persuaso Luigi ad andarsene.

Quando il verbo è nella forma riflessiva, tuttavia, quest'ultima distinzione risulta meno marcata, in quanto non è possibile avere dubbi sul soggetto dell'infinito.

5. Gianni si persuase ad andarsene.

In conclusione, abbiamo visto come la pronuncia corretta dei due verbi in questione sia quella piana, che è poi un retaggio della loro forma latina. Inoltre, abbiamo osservato come le reggenze dei due verbi siano diverse e siano variate nel tempo le preposizioni che introducono i loro complementi e come sia importante oggi prestare attenzione a quale è usata: nel caso di persuadere, in particolare, la preposizione potrebbe farvi capire se qualcuno vi ama o se invece è solo intenzionato a farlo in futuro. 
Nota bibliografica:

- Edoardo Lombardi Vallauri, Semantic and syntactic variation with Italian infinitives introduced by di and a: a preliminary recognition, in Mara Frascarelli (ed.), Structures and Meanings: CrossTheoretical Perspectives, Paris-Torino, L'Harmattan, 20II, pp. 38-60.

- Gunver Skytte, Giampaolo Salvi, Maria Rita Manzini, Frasi subordinate allinfinito, in Lorenzo Renzi (a cura di), Grande grammatica italiana di consultazione, Bologna, Il Mulino, I99I, pp. 483569 .

- Gunver Skytte, La sintassi dellinfinito in italiano moderno, Museum Tusculanum Press, I983.

\section{Cita come:}

Rossella Varvara, Vi vogliamo dissuadere o persuadere?, "Italiano digitale", 2019, IX, 2019/2 (aprile-giugno)

DOI: 10.35948/2532-9006/2020.3111

\section{Copyright 2019 Accademia della Crusca}

Pubblicato con licenza creative commons CC BY-NC-ND 